http://dx.doi.org/10.35381/racji.v5i1.598

\title{
La Educación Superior en el Ecuador: Derecho o servicio
}

\section{The Higher Education in Ecuador: Right or service}

\author{
María Gabriela Reyes-Idrovo \\ mreyesi@ucacue.edu.ec \\ Universidad Católica de Cuenca, Cuenca \\ Ecuador \\ https://orcid.org/0000-0003-2125-1928 \\ Cecilia Ivonne Narváez-Zurita \\ narvaez@ucacue.edu.ec \\ Universidad Católica de Cuenca, Cuenca \\ Ecuador \\ https://orcid.org/0000-0002-7437-9880 \\ Enrique Eugenio Pozo-Cabrera \\ epozoc@ucacue.edu.ec \\ Universidad Católica de Cuenca, Cuenca \\ Ecuador \\ https://orcid.org/0000-0003-4980-6403 \\ Juan Carlos Erazo-Álvarez \\ jcerazo@ucacue.edu.ec \\ Universidad Católica de Cuenca, Cuenca \\ Ecuador \\ https://orcid.org/0000-0001-6480-2270
}

Recibido: 15 de noviembre de 2019 Aprobado: 16 de diciembre de 2019

\section{RESUMEN}

La educación superior en el Ecuador ha sido constante tema de debate, a propósito de la Constituyente del año 2008, se presenta como un derecho evolutivo, que recibe las características del servicio público y se vuelve más exigible para todo ciudadano ecuatoriano o residente. La denominación de autonomía se ha entregado varias responsabilidades a los actores del sistema educativo superior. La universidad reclama 
libertad de autogestión, exige libertades políticas, y por sobre todo aclama liberación estatal. El ejercicio de la autonomía para las IES va a verse reflejado en la responsabilidad académica; pues sus actores no pueden responder si su desarrollo depende de acciones exógenas. En la presente investigación se traza como objetivo, determinar las formas de retribución de los actores del sistema de educación superior frente a sus responsabilidades, para ello se ha empleado los métodos analítico sintético e histórico lógico, con un enfoque cualitativo y un alcance descriptivo.

Descriptores: Derecho a la educación superior; Servicio; Autonomía; Responsabilidad académica.

\section{ABSTRACT}

Higher education in Ecuador has been a constant topic of debate, regarding the Constituent of the year 2008, it is presented as an evolutionary right, which receives the characteristics of the public service and becomes more enforceable for every Ecuadorian citizen or resident. The denomination of autonomy has given several responsibilities to the actors of the higher education system. The university demands freedom of self-management, demands political freedoms, and above all acclaims state liberation. The exercise of autonomy for HEls will be reflected in academic responsibility; because its actors cannot respond if their development depends on exogenous actions. In the present investigation, the objective is to determine the forms of remuneration of the actors of the higher education system against their responsibilities, for this the synthetic and historical analytical methods have been used, with a qualitative approach and a descriptive scope.

Descriptors: Right to higher education; Service; Autonomy; Academic responsibility.

\section{INTRODUCCIÓN}

Está cada vez más instituido el uso del Derecho Constitucional, al ser considerado como el área que da soporte a los derechos especializados, con origen en la atención de problemas de desigualdad y violencia que viven los países de Latinoamérica, además que aparece como resultado de fenómenos que lo han transformado como la internacionalización y la participación activa del juez garantista que, invadido de los instrumentos internacionales va poniendo en práctica las normas de rango 
constitucional ante las necesidades de sus pueblos (Correa, Osuna, y Ramírez, 2017). Cuando se presentan problemas jurídicos que requieren una consideración y análisis superior, entonces entra en acción el Derecho Constitucional.

Esta rama del Derecho Público ha recibido considerable atención crítica desde el nacimiento de los sistemas internacionales de derechos humanos, lo que ha generado la necesidad de que los países construyan nuevas constituciones, en el caso ecuatoriano desde octubre del año 2008, se encienden las alertas de estudiar, entender y practicar el neoconstitucionalismo como una corriente que está llena de características que se evidencian de acuerdo a las connotaciones que la situación ofrece, es decir, puede ser un neoconstitucionalismo ontológico, epistemológico o político - social (Zavala, 2010).

El Ecuador es un Estado constitucional de derechos, que en su Carta Magna ha enumerado entre sus deberes garantizar sin discriminación alguna el goce de los derechos de rango constitucional (Asamblea Constituyente, 2008). Dicho de otro modo, la Constitución ecuatoriana es un catálogo de derechos, que se obliga por sí sola a cumplirlos y hacerlos cumplir. Lo antes expuesto, lleva a encontrar que entre los derechos fundamentales que se ciñen en la constitución está el derecho a la educación, por lo que, se analizará la educación superior en el Ecuador, para saber si es un derecho, o si puede obtener las características de un servicio, con las corresponsabilidades que esto conlleva.

No hay un acuerdo general respecto a que la educación superior en el Ecuador, en un primer momento sea vista como servicio, que debe estar en alineación directa a los parámetros de calidad establecidos por el organismo de control, que se denomina Consejo de Aseguramiento de la Calidad de la Educación Superior (CACES). En el Ecuador el servicio público de educación, se presta a través de Instituciones de Educación Superior (IES) Públicas, Privadas y Privadas con financiamiento estatal a las que se les denomina cofinanciadas, aunque esa clasificación es para otra connotación, la calidad del servicio es medida en cada una de estas IES, pues como todo servicio es 
susceptible de mediciones cualitativas y cuantitativas, y así mismo, debe ser prestado de manera amplia no monopólica y mantener estándares del servicio público.

Se ha prestado escasa atención, en que la educación superior como un derecho fundamental del ser humano, debe tener como finalidad la vinculación, pues el derecho no es sólo a educarse sino a relacionarse e interactuar con la comunidad a través de proyectos sostenibles que generen soluciones a los problemas sociales, establecidos mediante los resultados previos de las investigaciones científicas realizadas.

Entonces, desde la óptica de la educación superior como derecho, se ha determinado que está compuesto de objetivos y principios, como el de la vinculación que es función esencial de la educación, ya que a partir de la vinculación los actores de la educación devuelven respuestas a las necesidades de la sociedad, por consiguiente, debe existir una obligación de los actores del sistema, es decir, tanto del Estado y sus delegatarios como de los estudiantes. En este sentido, la presente investigación tiene por objetivo establecer los valores de responsabilidad y compromiso académico de los actores del sistema de educación superior, para ello es necesario analizar la Constitución, la Ley Orgánica de Educación Superior y el Reglamento de Régimen Académico del Consejo de Educación Superior, así como las concepciones de la educación superior en América Latina, y a través de este análisis aportar a los criterios de responsabilidad académica.

\section{DESARROLLO}

\section{Derecho a la educación y a la educación superior}

Sobre el derecho a la educación se encuentran varias acepciones, sin embargo, es un tema que ha tenido mayor estudio y desarrollo en España, donde se ha partido de una visión general hasta llegar a la constitucionalización del mismo, a partir del año 1812 se le dio una visión prestacional. En el siglo XX la prestación de la educación toma mayor fuerza y manteniendo la dimensión constitucionalista, se alista a desarrollar este servicio en la Constitución de Cádiz, donde no solo se contempló la educación escolar 
sino la superior y otras instrucciones, además se puso en marcha la gratuidad de todos los niveles educativos a pesar de que, después se modificó con decretos reales y leyes. Así era considerada la educación como derecho a recibir instrucción y que estaba conectada a la necesidad y posición de quienes escasean de instrucción, que es quien pasa a ser el titular del derecho a la educación exigiendo su prestación al Estado (Vidal, 2017).

En Ecuador, la educación superior presenta cambios imprescindibles en la última década, y con las reformas a su ley, se puede ver que la situación implica múltiples requerimientos (ver figura1), por lo que para cumplir los retos de la educación es necesario partir tomando en cuenta las expectativas de las universidades en el mundo y de los consumidores de este servicio. Cuando se dice que la educación es un servicio público, es común relacionarlo con la gratuidad y el mal servicio; sin embargo, a la educación superior impartida en las universidades públicas, no se la ha considerado en su contexto general, una situación es la calidad de la enseñanza y otra el servicio de atención, que comprende desde el acceso al propio sistema hasta la titulación, mientras que en las universidades particulares lo que más se ha destacado es el servicio en: atención, infraestructura, acceso y la simplificación en trámites, es decir, han existido diferencias en la medición del servicio. Al respecto, los autores Tobón y otros (como se citó en González, Vélez, y Méndez, 2018) señalan que:

En la educación superior se considera consumidor al estudiante, mismo que cumplirá con ciertas obligaciones en el transcurso de su carrera universitaria, detalladas en los reglamentos de la institución educativa; mientras que el proveedor, es la entidad universitaria quien brindará los servicios de educación superior, siendo éstos de calidad (p. 103).

Como consta en líneas anteriores, es importante definir que en la educación superior como servicio coexisten dos partes, una primera que es el estudiante quien ocupa el rol de consumidor de la educación y el Estado prestador del servicio de manera directa o indirecta con sus delegatarios, y en esta relación de ida y vuelta ambos denotan la calidad en su rol. En definitiva, la educación superior no abandona las características de 
un bien público social y por tanto un bien de consumo, lo que exige de las dos partes una participación responsable, y se materializa una relación de dependencia existencial, no puede estar el prestador sin el consumidor o viceversa, aunque en la actualidad en Ecuador, el prestador no cubre la demanda.

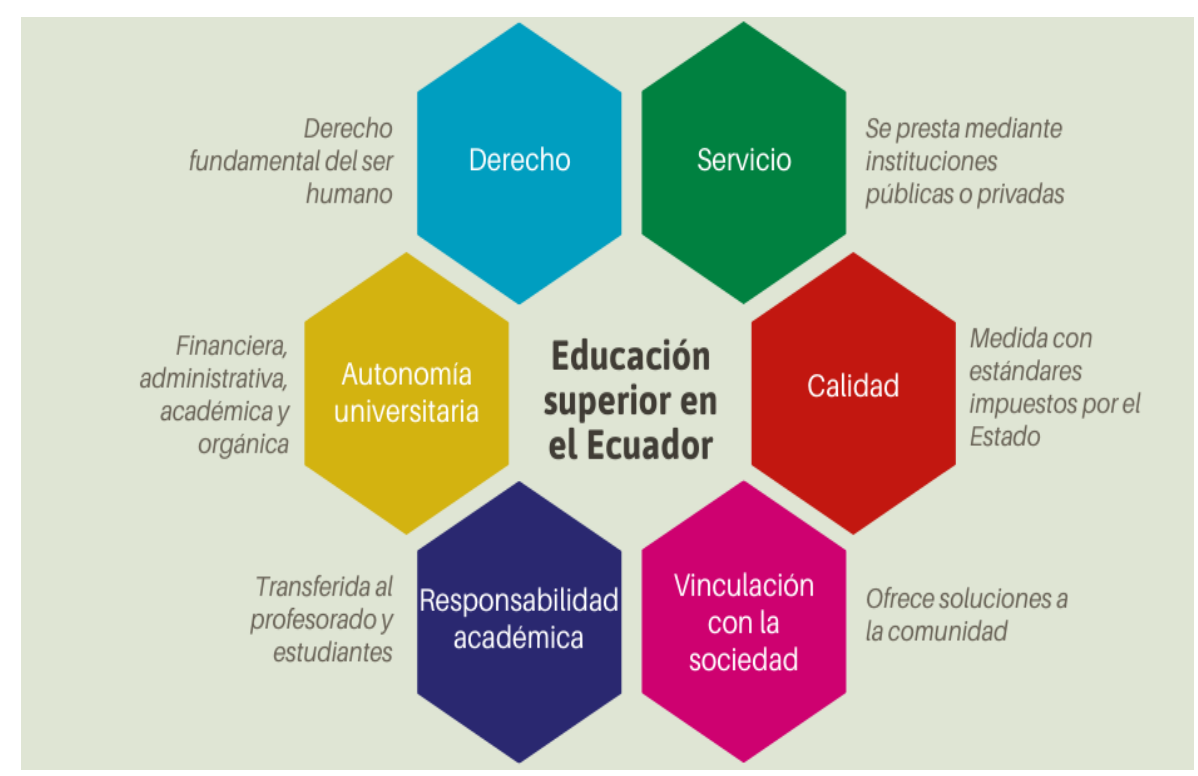

Figura 1. Educación Superior en el Ecuador.

Retomando el tema central, la educación superior como derecho va relacionado a otros derechos sociales, que hacen posible el ejercicio del derecho a la cultura, derecho al disfrute de los avances de la ciencia y la tecnología, así como el derecho a la investigación y producción científica. Como lo ha señalado Pozo (2016), la autonomía de la voluntad es parte del derecho a la dignidad de la persona, teniendo así la oportunidad de ejercer la autodeterminación y consecuentemente la libertad de planificar y proyectar un futuro en base a la investigación.

En lo referente a que el derecho a la educación superior se lo considera como un servicio, y que; por tanto, es exigible una contraprestación por parte del cliente, la producción científica es otro de los mecanismos de devolución y responsabilidad académica que puede y debe utilizar el estudiante, producir es su derecho y también se 
vuelve una obligación, pues con las herramientas que la universidad le ha proporcionado está en capacidad de ofrecer productos de sus investigaciones (Aldana Zavala, 2019).. Por ello, los temas de investigación que aportarán seriamente a la solución de problemas de la sociedad son los temas de las ciencias sociales, que atiendan la pobreza y las desigualdades sociales, sin descuidar las investigaciones en beneficio de la salud pública (Bunge, 2019).

Ante todo, se debe recordar que la educación superior sea considerada derecho o servicio, siempre apuntará a la invaluable labor de brindar soluciones a los problemas de la sociedad, y esto se lo hace a través de la presentación y ejecución de proyectos, de ahí que con la investigación se determinarán los verdaderos problemas y se plasmarán las soluciones más oportunas para mejorar la calidad de vida de una población determinada. Mientras que, al ser un bien público obtiene inmediatamente el carácter de social, por ello las exigencias en la mejora continua a su acceso, con la finalidad de que sea un derecho subjetivo real, social y exigible al Estado consignatario, que el proveedor lo incluya dentro de su estructura política como un derecho primario, que su exigencia no se encuentre con la desilusión de desarrollarse de acuerdo al modelo constitucional estatal débil en la protección de un derecho social (Morales, 2015).

La educación superior también es un bien público. Esto es debatible en muchos países de Latinoamérica, pues siempre desde el punto de vista político representa números, a causa de que interviene directamente en el presupuesto anual de un Estado, y el gasto público siempre se verá directamente afectado o inflado con este rubro de obligación social. Por tanto, la educación superior, es parte de los derechos sociales que tienen una dimensión prestacional vinculada a la disponibilidad financiera y política de los Estados, comparable con el derecho a la salud, que siempre será dependiente de los presupuestos estatales (Guanipa Ramírez, Albites Sanabria, Aldana Zavala \& Colina Ysea, 2019). 
Sin duda, el acceso al sistema de educación superior es la barrera más difícil de romper, las diferencias económicas y culturales son determinantes para que una parte de la ciudadanía no mida las consecuencias de estancarse en su proceso de formación, la problemática en el ejercicio de este derecho, se encuentra en la característica de servicio social que se le ha dado, pues la forma de presentación de la educación tiene particularidades como el acceso, la continuidad, permanencia y una etapa final que es en este caso el alcance de una satisfacción en su resultado, que se mide únicamente con la entrega de profesiones certificadas (Villar, 1979).

Con más detenimiento en este aspecto, la educación en versión derecho - servicio público, es brindado de manera propia o impropia, esto quiere decir que se ofrece en las IES públicas o privadas; y sin importar aquello la finalidad siempre será el brindar un servicio de calidad para la sociedad, lo que en este espacio es el estudianteconsumidor quien, al recibir la prestación educacional, debe retribuir la inversión estatal o de fondos propios a través de la responsabilidad académica - social, porque lo que recibe como servicio debe tener la manera de retribución establecida, y ahora el modelo educacional ecuatoriano implanta dentro de las funciones sustantivas para la consecución de los fines de la educación superior: docencia, investigación y vinculación, esta última está para generar intercambios de conocimientos, de planes, proyectos, programas que contribuyan al medio ambiente y al desarrollo productivo (Ley Orgánica de Educación Superior, 2019).

Al ser un derecho la educación superior gratuita, es necesario que exista una contraprestación moral, de responsabilidad social, con la finalidad de que el Estado proveedor, pueda conseguir como logro ante tal inversión un mejor desarrollo de su pueblo, al momento en que éste es educado sin la preocupación económica de sostener sus estudios. Es decir que, con la gratuidad y democratización de la educación superior, nace la responsabilidad académica como parte de la retribución de la sociedad beneficiaria (Pazos, 2018). 
Especialmente, se debe tener claro que la educación siendo un derecho no deja de ser un servicio, y como tal debe contemplar entre otras características a la calidad y mejora continua, lo que no deslinda al estudiante el ser parte activa y fundamental para lograr este objetivo, a través de su participación progresiva en actividades que, siendo extracurriculares, formen parte del proceso de enseñanza - aprendizaje y que estén destinadas para brindar un efectivo servicio de la comunidad.

El impacto que ha tenido la educación superior y su internacionalización ha generado consecuencias comerciales que se sintieron en la comunidad académica a partir del año 2001, cuando distintas asociaciones, americanas y europeas, firmaron la declaración conjunta, en contra del Acuerdo General sobre el Comercio de Servicios (GATS) por haber incluido a la educación superior como uno de los servicios sujetos a negociación, lo que provocó que varias ONGs, impulsen programas en contra de esta concepción de la educación superior como servicio (Guarga, 2008).

Como conclusión derivada de lo expuesto, es necesario indicar que las responsabilidades deben ir de la mano tanto para los prestadores del servicio de educación superior cuanto del consumidor, que es el estudiante. Quien recibe educación de calidad va a brindar siempre una devolución responsable y acorde a los problemas sociales, buscando de manera permanente las soluciones a las situaciones económicas, productivas, emprendedoras, sociales, políticas, entre otras, que se le presenten en su entorno y lo va a hacer de manera coordinada con su proveedor del servicio educacional que es la universidad.

\section{Autonomía universitaria en el Ecuador}

En el caso ecuatoriano, la educación superior está apegada a principios, tales como universalidad, igualdad, equidad, progresividad, interculturalidad, solidaridad y no discriminación, así mismo se establecen varios criterios para su desarrollo, entre ellos el de responsabilidad, por tanto, este criterio anclado en los principios es obligatorio para los sujetos, es decir, tanto para las IES como para los estudiantes. 
La autonomía universitaria ha cobrado importancia a la luz de la reciente Ley Orgánica de Educación Superior (LOES), que cada vez se ajusta más a la realidad de la educación superior en el mundo; el camino a seguir para las IES es la autonomía universitaria, y esto en el Ecuador se presentó desde la Constitución de1945, siempre ha sido un tema rodeado de protesta y exigencia social, producto de ello, con los años se ha ido mejorando su prestación, hasta que el Constituyente recoge todas las reclamaciones de los sectores y en la Constitución del año 2008 declara a la educación como un servicio público, y al asumirlo como un bien público asume la rectoría del sistema educativo nacional, para que se creen políticas educacionales, y establece su regulación y constante evaluación.

Actualmente, uno de los debates más significativos en el derechos a la educación son los principios que cobijan a la educación superior y así se encuentra el de la autonomía, consagrado en la constitución y desarrollado en la LOES, la autonomía de la que se les atribuye a las universidades es financiera, académica, administrativa y orgánica, el ejercicio de este principio va de la mano con los principios de equidad, solidaridad, justicia, participación ciudadana y responsabilidad social, entre otros (Ley Orgánica de Educación Superior, 2018). El ejercicio de la autonomía por parte de las universidades debe ser responsable, y no solo que es para la universidad como institución, sino que se extiende a sus profesores e investigadores para la libertad de cátedra e investigación.

La autonomía orgánica se materializa en la libertad de expedir normativa interna que considera cada IES, claro siempre en concordancia con las leyes aplicables a la materia; la autonomía académica, es el albedrío que tiene cada universidad o instituto de educación superior en la elaboración de sus programas de estudios; la autonomía administrativa permite que las IES nombren a sus autoridades, docentes, investigadores y trabajadores, además está dentro de esta autonomía la creación o reorganización de procesos internos y la determinación de las formas de gobierno. La autonomía financiera de la que gozan las universidades e institutos superiores se 
refiere a la elaboración y aprobación de un presupuesto institucional anual y su ejecución es puesta a conocimiento de la sociedad mediante una rendición de cuentas, así mismo tiene libertad en la administración patrimonial.

La autonomía ha recibido considerable atención crítica desde las reformas legales, donde se ha visto que través de estas libertades, las universidades e institutos superiores tienen la obligación de ampliar sus servicios, para que estos sean medidos bajo estándares de calidad, y de ese modo el ente estatal califique y cualifique los servicios educacionales prestados por los miembros del sistema de educación superior nacional. La evidencia reciente sobre la materia sugiere que la autonomía es la permisibilidad que les entrega o autoriza el Estado a las IES; sin embargo, se encuentra que a la autonomía universitaria la han definido como autonomía responsable y como lo indica Rojas (2011) el que se haya incluido el término responsable en el texto legal es para delimitar las posibles confusiones de la autonomía entregada.

Al día de hoy, continúa el debate sobre la autonomía delimitada como responsable, que ha sido dada como un permiso limitado para que las universidades puedan hacer lo que el Estado les permita y cuando a través de los mecanismos utilizados para medir la calidad educativa se encuentra algo no conveniente se les castigue con calificaciones o puntajes por debajo de lo esperado, ubicando a las instituciones educativas en una espera angustiosa, esto sólo cuando se trata de temas que entran en los famosos indicadores de calidad. No obstante, otra cosa es la autonomía fiscalizada por el organismo de control estatal Consejo de Educación Superior (CES) que tiene declarada en su misión planificar, coordinar y regular el sistema de educación superior nacional, así como ser el canal entre los actores del sistema y la función ejecutiva (Consejo de Educación Superior, 2016).

Con las reformas legislativas y la promulgación de la autonomía como principio regente del sistema de educación superior, las intervenciones estatales van siendo más disimuladas; sin embargo, existen nuevas formas de tener cierta injerencia en las universidades e institutos superiores, como la asignación presupuestaria y los procesos 
de acreditación de fondos, entre otros (Barreto, 2015). Los cambios siempre se dan para mejorar algo determinado, en el caso de la educación superior que históricamente ha estado envuelta de personajes políticos, que han utilizado a la academia como su oportunidad para generar caos, contraparte, inestabilidad en la gobernabilidad del Estado, pocos han visto a este sector como un semillero de líderes; como el medio de ofrecer un mejor futuro al país, intentando hacer creer que las IES son sólo fábrica de títulos, no han querido fortalecer el pensamiento crítico, la producción científica, o la vinculación con la sociedad a través de productos que las universidades, escuelas politécnicas e institutos superiores tienen para brindar soluciones a los problemas sociales.

Quienes han elevado su protesta en favor de la autonomía universitaria han dejado claro que su preocupación siempre ha sido que con las intervenciones estatales se menoscabe la autonomía, que ésta sea sólo un enunciado, dando paso a que los actores reclamen en las calles la capacidad de las universidades para autogobernarse, permitiendo la democratización de las IES, y anulando toda posibilidad de privatización o corporativismo del derecho a la educación superior (Eguiguren, 2017). Es importante recalcar que la autonomía con la última reforma a la LOES ha sido ampliada y se deja la puerta abierta a una cercana auto gobernabilidad donde las asignaciones presupuestales provenientes del Estado tal vez sean la única relación Estadouniversidad.

Se ha prestado escasa atención a la realidad de las universidades públicas, tal vez es un poco más complejo alcanzar la autonomía plena, necesitan replantearse desde sus bases, pues sus formas de gobierno y administración son netamente políticas y dependientes del gobierno de turno, y todo ello, aunque parezca increíble influencia profundamente en el quehacer académico, por eso hay pensadores que estiman que la rehumanización debe volver a la cuna de la educación pública, cultivando así la responsabilidad con el entorno (León, 2013). 
Concluyendo esta sección, se infiere que la autonomía universitaria debe ser entendida como la libertad para que las instituciones de educación superior, llámense universidades, escuelas politécnicas, institutos superiores o conservatorios; ejerzan su administración de manera tal que no tengan cortapisas en su autogestión, el financiamiento estatal es necesario, sin duda; la asignación presupuestaria no es un favor a las IES es un deber que el Estado tiene con la población estudiantil, para cumplir su mandato de gratuidad, tiene que entregar recursos suficientes para la formación de sus generaciones, así como mantener la subvención a las IES particulares llamadas cofinanciadas por recibir recursos estatales que cubren únicamente el costo por estudiante al que el Estado le ha asignado beca por política de cuotas, al respecto, hay críticos que conciben al cofinanciamiento como el pago de las cuentas de la universidad privada, cuando en realidad se cubre el gasto operacional que implica el incremento de estudiantes becados por el Estado.

\section{Responsabilidad académica}

Desde el inicio, en el presente trabajo se hace referencia a la responsabilidad académica, pues a pretexto de derecho exigible, por estar constitucionalmente garantizado, el derecho a la educación se presta a través de un servicio público, justo por esta característica los beneficiarios están socialmente obligados a reciprocar lo entregado por el Estado en materia de educación, y por eso las formas de participación estudiantil han ido evolucionando, para ser hoy en día actores y gestores de nuevas corrientes e ideas, generando propuestas y obteniendo logros para otros ciudadanos. La educación superior se ha empezado a ofertar en varias regiones del mundo por distintas instituciones educativas, que se vuelven proveedores y que no atienden la necesidad educacional, como modo de servir a la sociedad ávida de conocimientos y herramientas para mejorar su calidad de vida pues, lo ofrecen como un servicio, comparable a franquicias comerciales en las que no cuenta el derecho sino el servicio, 
es más, por ello también se da la oferta de otras modalidades de estudios (García, 2005).

Es importante definir qué es la responsabilidad, y como lo señala el Diccionario Jurídico Anbar, responsabilidad es la capacidad de obligarse a responder por actos propios (Fondo de Cultura Ecuatoriana, 1999), mientras que, para el profesor Zavala (2014), la responsabilidad se vuelve un juicio de valor, donde el responsable confronta a una norma escrita o moral por su incumplimiento, por su parte, el constitucionalista Pozo (2015), en su obra sobre derecho constitucional, refiere que los derechos implican permisiones y prohibiciones, entonces, las responsabilidades son obligatorias y tienen carácter de exigibles y determinantes a un grupo de beneficiarios de tal o cual derecho, por tanto, el derecho a la educación tiene su retribución por parte de quienes reciben el servicio, y es cumplir con sus obligaciones directas con el Estado y la universidad.

Los actores de la educación superior llámese profesores, investigadores y estudiantes tiene que ejercer la responsabilidad académica, los primeros en el cumplimiento de su cátedra abierta libre de pensamientos que influyan en sus educandos, sin tipologías amorfas y que desnaturalicen el actuar del docente experto en su área del conocimiento; el investigador, cumpliendo su parte devolutiva a la sociedad mediante propuestas salvadoras a la realidad con propósitos positivos que signifiquen cambios, no revisiones de temas ya analizados sino análisis que arrojen resultados de evolución; $y$, por otra parte, la responsabilidad del estudiante, quien debe responder por el servicio que recibe, el Estado invierte en él y la sociedad merece recibir su producción, requiere conocer lo que éste puede hacer por cambiar la realidad social, así surge la inclusión de trabajos finales integrales en las mallas de los programas académicos, o las horas de vinculación con la sociedad que debe cumplir un estudiante para culminar su carrera.

En este trabajo se ha analizado el papel del docente quien también tiene responsabilidades que son medidas y que deben presentarse como devolución para que la calidad del servicio alcance la excelencia. Al profesorado se le ha entregado un conglomerado de responsabilidades que son determinantes para mejorar los 
compromisos académicos, tiene a su cargo la elaboración de los programas, de asesoría, sugerir aspectos metodológicos entre otros (Villa, 2002). La carga docente sin duda es extenuante y llena de retos, el proceso de enseñanza empieza desde la planificación y culmina sólo con la satisfacción del profesorado al ver a sus estudiantes despuntar y ofrecer soluciones a la comunidad, por eso y más la labor docente es de gran reconocimiento, por la maleta de responsabilidades que lleva, incluso la responsabilidad social y emocional.

La responsabilidad estudiantil va direccionada a que el estudiante asuma su aprendizaje en todos los niveles y con todas las implicaciones que el proceso de formación conlleva (García, Fonseca, y Concha, 2015) La vinculación se posiciona cada vez más como un gran ejemplo de como el cliente de este servicio educacional va aprendiendo a generar respuestas a su comunidad, aunando esfuerzos entre carreras para crear proyectos beneficiosos que cambian la vida de los participantes, es esta una de las formas de demostrar la responsabilidad académica, no obstante, la responsabilidad también se mide en los logros académicos, en el rendimiento de cada uno de los estudiantes al cumplir con las exigencias que les impone cada asignatura.

\section{METODOLOGÍA}

Para la construcción de este trabajo de investigación han sido utilizados los métodos investigativos (Palella y Martins, 2012) analítico-sintético con enfoque cualitativo por que condujo la investigación a la revisión técnica documental de artículos y libros de estudiosos proponentes nacionales e internacionales del derecho a la educación superior, quienes emiten opinión viendo a la educación como un servicio que es exigible como derecho fundamental; el texto se ha enriquecido con el método histórico-lógico, con el que se analizó la historicidad de la educación, origen y evolución, encontrando la mejora continua en el sistema de educación superior ecuatoriano, así como la autonomía reconocida a las universidades y como resultado la determinación de reglas para el ejercicio de la responsabilidad académica (Zamora, Narváez y Erazo, 2019). 


\section{APORTES}

El reconocimiento y la puesta en práctica de la educación superior como derecho y que a la vez haya sido categorizada como servicio público, denotan que la universidad pública o privada cumple un servicio público a través de los componentes sistemáticos como la docencia, investigación y la vinculación (Cotino, 2012).

La educación vista como un derecho fundamental del ser humano, debe tener como aplicación directa la vinculación, pues el derecho no es sólo a educarse sino a relacionarse e interactuar con la comunidad a través de proyectos sostenibles que generen soluciones a los problemas sociales, establecidos mediante los resultados de las investigaciones científicas realizadas previamente.

Sin embargo, otros aspectos quedan no inscritos en este trabajo, por lo que serán objeto de un estudio más integral, para continuar abonando a la importancia de que el derecho a la educación se lo preste con la calidad de un servicio público, y que sus prestadores mantengan autonomía en la gobernabilidad y administración, siempre sin confundirlo con libertinaje de poder, y que los actores del sistema asuman la responsabilidad que en cada medida les sea asignada.

\section{REFERENCIAS CONSULTADAS}

1. Asamblea Constituyente. (2008). Constitución de la República del Ecuador. Montecristi, Ecuador: Asamblea Constituyente.

2. Aldana Zavala, J. J. (2019). La competencia epistemológica en el investigador social universitario venezolano. Praxis, 15(1), 103-115. https://doi.org/10.21676/23897856.3091

3. Barreto, D. (2015). La autonomía universitaria en el Ecuador. Revista Anales, 1(373), 255.

4. Bunge, M. (2019). Investigación científica: enfoque, método y evaluación. Lima, Perú: Fondo Editorial. 
5. Consejo de Educación Superior. (01 de Junio de 2016). Estatuto Orgánico por Procesos del Consejo de Educación Superior. RPC-SO-21-No.335-2016. Quito, Pichincha, Ecuador.

6. Correa, M., Osuna, N., y Ramírez, G. (2017). Lecciones del Derecho Constitucional (Vol. 1). Bogotá, Colombia: Universidad Externado de Colombia.

7. Cotino, L. (2012). El derecho a la educación como derecho fundamental. Especial atención a su dimensión social prestacional. Madrid, España: Centro de Estudios Políticos y Constitucionales.

8. Eguiguren, C. (2017). La autonomía universitaria en Ecuador tras una década de la Revolución Ciudadana. Revista de Derecho UNED(21), 322.

9. Fondo de Cultura Ecuatoriana. (1999). Diccionario Jurídico Anbar con Legislación Ecuatoriana. Cuenca, Azuay, Ecuador: Fondo de Cultura Ecuatoriana.

10. García, C. (2005). Complejidades de la globalización e internacionalización de la educación superior Interrogantes para América Latina. Cuadernos del CENDES, 6.

11. García, F., Fonseca, G., y Concha, L. (2015). Aprendizaje y Rendimiento Académico en Educación Superior: Un Estudio Comparado. Revista Electrónica "Actualidades Investigativas en Educación".

12. González, D., Vélez , S., y Méndez, S. (2018). Las exigencias de la calidad en la educación universitaria y la aplicabilidad de los derechos del consumidor: análisis actual y propuesta de mejora. Killkana Social, 2(4), 104.

13. Guarga, R. (2008). La educación superior y los acuerdos de la Organización Mundial de Comercio. Universidades(38), 11.

14. Guanipa Ramírez, L., Albites Sanabria, J., Aldana Zavala, J., \& Colina Ysea, F. (2019). Educación para la ciudadanía y la democracia: El equilibrio del poder. IUSTITIA SOCIALIS, 4(6), 71-89. doi:http://dx.doi.org/10.35381/racji.v4i6.290

15. León, C. (Diciembre de 2013). Cambiar el alma... ¿Exiliar a las humanidades? Ecuador Debate, 90, 100.

16. Ley Orgánica de Educación Superior. (02 de Agosto de 2018). Quito, Ecuador: Corporación de Estudios.

17. Morales, L. (2015). Derechos sociales constitucionales y democracia. Madrid, España: MARCIAL PONS.

18. Palella, S., y Martins, F. (2012). Metodología de la Investigación Cuantitativa. Caracas, Venezuela: FEDUPEL. 
19. Pazos, R. (2018). Acceso a la educación superior: Uruguay y Ecuador Estudio de derecho comparado. UNIVERSITAS.

20. Pozo, E. (2015). Derecho Procesal Constitucional. Cuenca, Ecuador: EDÚNICA.

21.Pozo, E. (Enero de 2016). El derecho de investigar y el deber de difundir lo investigado. ODONTOLOGÍA activa UCACUE, 1(1), 81.

22. Rojas, J. (2011). Reforma universitaria en el Ecuador, Etapa de transición. Innovación Educativa, 11(57), 62.

23. Vidal, C. (2017). El derecho a la educación en España. Madrid, España: Marcial Pons Ediciones Jurídicas y Sociales, S.A.

24. Villa, M. (2002). Flexibilidad y Educación Superior en Colombia. Bogotá, Colombia: Instituto Colombiano Para el Fomento y Desarrollo de la Educación Superior.

25. Villar, J. (1979). El derecho a la educación como servicio público. (C. d. Constitucionales, Ed.) Administración Pública(88), 158.

26.Zamora Cabrera, E. P., Narváez Zurita, C. I., \& Erazo Álvarez, J. C. (2019). Incidencia del control interno en la gestión administrativa de las IES. Caso: Departamento de Pastoral, Universidad Politécnica Salesiana. Koinonía, 339.

27.Zavala, J. (2010). Derecho constitucional, neoconstitucionalismo y argumentación jurídica. Guayaquil, Ecuador: Edilex S.A.

28. Zavala, J. (2014). Responsabilidad penal de las personas jurídicas en el sistema constitucional ecuatoriano (arts. 49 y 50 coip): una guía teórica - práctica. Samborondón, Ecuador: Universidad Espíritu Santo. 